Louisiana State University

LSU Digital Commons

Faculty Publications

Department of Physics \& Astronomy

$1-1-2008$

\title{
The final spin from the coalescence of aligned-spin black hole binaries
}

\author{
Luciano Rezzolla \\ Max Planck Institute for Gravitational Physics (Albert Einstein Institute) \\ Peter Diener \\ Louisiana State University \\ Ernst Nils Dorband \\ Louisiana State University \\ Denis Pollney \\ Max Planck Institute for Gravitational Physics (Albert Einstein Institute) \\ Christian Reisswig \\ Max Planck Institute for Gravitational Physics (Albert Einstein Institute)
}

See next page for additional authors

Follow this and additional works at: https://digitalcommons.Isu.edu/physics_astronomy_pubs

\section{Recommended Citation}

Rezzolla, L., Diener, P., Dorband, E., Pollney, D., Reisswig, C., Schnetter, E., \& Seiler, J. (2008). The final spin from the coalescence of aligned-spin black hole binaries. Astrophysical Journal, 674 (1 PART 2)

https://doi.org/10.1086/528935

This Article is brought to you for free and open access by the Department of Physics \& Astronomy at LSU Digital Commons. It has been accepted for inclusion in Faculty Publications by an authorized administrator of LSU Digital Commons. For more information, please contact ir@lsu.edu. 
Authors

Luciano Rezzolla, Peter Diener, Ernst Nils Dorband, Denis Pollney, Christian Reisswig, Erik Schnetter, and Jennifer Seiler

This article is available at LSU Digital Commons: https://digitalcommons.Isu.edu/physics_astronomy_pubs/1445 


\title{
THE FINAL SPIN FROM THE COALESCENCE OF ALIGNED-SPIN BLACK HOLE BINARIES

\author{
Luciano Rezzolla, ${ }^{1,2}$ Peter Diener, ${ }^{2,3}$ Ernst Nils Dorband, ${ }^{1}$ Denis Pollney, \\ Christian Reisswig, ${ }^{1}$ Erik Schnetter, ${ }^{2,3}$ AND Jennifer Seiler ${ }^{1}$ \\ Received 2007 October 18; accepted 2007 December 21; published 2008 January 29
}

\begin{abstract}
Determining the final spin of a black hole $(\mathrm{BH})$ binary is a question of key importance in astrophysics. Modeling this quantity in general is made difficult by the fact that it depends on the seven-dimensional space of parameters characterizing the two initial black holes. However, in special cases, when symmetries can be exploited, the description can become simpler. For BH binaries with unequal masses but with equal spins which are aligned with the orbital angular momentum, we show that the use of recent simulations and basic but exact constraints derived from the extreme mass-ratio limit allow us to model this quantity with a simple analytic expression. Despite the simple dependence, the expression models very accurately all of the available estimates, with errors of a couple of percent at most. We also discuss how to use the fit to predict when a Schwarzschild $\mathrm{BH}$ is produced by the merger of two spinning BHs, when the total angular momentum of the spacetime "flips" sign, or under what conditions the final BH is "spun up" by the merger. Finally, we suggest an extension of the fit to include unequal-spin binaries, thus potentially providing a complete description of the final spin from the coalescence of generic BH binaries with spins aligned to the orbital angular momentum.
\end{abstract}

Subject headings: black hole physics — gravitational waves — relativity — stars: statistics

Online material: color figures

\section{INTRODUCTION}

The determination of the final spin of a BH binary is a question of key importance in astrophysics. Modeling this in general is made difficult by the fact that it depends on the seven-dimensional space of parameters characterizing the two initial BHs. However, in special cases, when symmetries can be exploited, the description can be much simpler.

Several recent studies have shed light on the remnant of the merger process. Using conservation principles, Hughes \& Blandford (2003) argued that mergers rarely lead to rapidly rotating objects. Gonzalez et al. (2007a) numerically evolved a sequence of nonspinning unequal-mass BHs, arriving at detailed estimates of the radiated energy and angular momentum. In a series of papers (Koppitz et al. 2007; Pollney et al. 2007; Rezzolla et al. 2007) we have studied the parameter space of mergers of equal-mass $\mathrm{BH}$ binaries whose spins are aligned with the orbital angular momentum but otherwise arbitrary. The findings agree well with independent numerical evolutions (Campanelli et al. 2007; Herrmann et al. 2007), as well as more recent studies of models with initial spins up to $J / M^{2}=0.8$ (Marronetti et al. 2007). An important result of these studies has been the determination of simple (quadratic) fitting formulas for the recoil velocity and spin of the merger remnant as a function of the initial BH parameters (Rezzolla et al. 2007).

A number of analytical approaches have been developed over the years to determine the final spin of a binary coalescence (Damour 2001; Buonanno \& Damour 2000; Buonanno et al. 2006; Damour \& Nagar 2007; Boyle et al. 2007). Very recently, an interesting method, inspired by the dynamics of a test particle around a Kerr BH, has been proposed for generic binaries (Buonanno et al. 2007a, hereafter BKL07). The approach as-

\footnotetext{
${ }^{1}$ Max-Planck-Institut für Gravitationsphysik, Albert-Einstein-Institut, 14476 Potsdam-Golm, Germany.

${ }^{2}$ Department of Physics and Astronomy, Louisiana State University, Baton Rouge, LA 70803.

${ }^{3}$ Center for Computation and Technology, Louisiana State University, Baton Rouge, LA 70803.
}

sumes that the angular momentum of the final $\mathrm{BH}$ is the sum of the individual spins and of the orbital angular momentum of a test particle on the last stable orbit of a Kerr BH with the same spin parameter as that of the final $\mathrm{BH}$.

Here we combine the data obtained in recent simulations to provide a phenomenological but analytic estimate of the final spin in a binary $\mathrm{BH}$ system with arbitrary mass ratio and spin ratio, but in which the spins are constrained to be parallel to the orbital angular momentum. Our numerical simulations have been carried out using the CCATIE code (Pollney et al. 2007). In addition to the data presented in Rezzolla et al. (2007) we add three simulations of equal-mass, high-spin binaries and three simulations of unequal-mass, spinning binaries (see Table 1). Other data are taken from unequal-mass, nonspinning binaries (Gonzalez et al. 2007a; Berti et al. 2007; Buonanno et al. 2007b) and from equal-mass, spinning binaries (Rezzolla et al. 2007; Marronetti et al. 2007); all of the Albert-EinsteinInstitut (AEI) data are summarized in Table 1. To avoid the possible contamination from the errors associated with highspin binaries reported by Marronetti et al. (2007) we have not considered binaries with initial spin $\left|J / M^{2}\right| \geq 0.75$ reported in the literature (Campanelli et al. 2007; Marronetti et al. 2007). We have, however, considered estimates of high-spin binaries (see Table 1), for which we know that the spins remain essentially constant prior to merger, with changes of less than $0.5 \%$ (Pollney et al. 2007), and that are very well captured by the fit.

\section{METHODS AND RESULTS}

We start by considering the final spin $a_{\text {fin }}$ as a function of the two free variables in the problem: the symmetric mass ratio $\nu \equiv M_{1} M_{2} /\left(M_{1}+M_{2}\right)^{2}$ and the spin of the initial BHs $a \equiv$ $J / M^{2}$, i.e., $a_{\text {fin }} \equiv J_{\text {fin }} / M_{\text {fin }}^{2}=a_{\text {fin }}(a, \nu)$. (Note that $a$ is dimensionless and not the angular momentum per unit mass.) By construction $a_{1}=a_{2}=a$ and $\boldsymbol{a} /|\boldsymbol{a}|= \pm \boldsymbol{L} /|\boldsymbol{L}|$, where $\boldsymbol{L}$ is 
TABLE 1

Initial Parameters of the New Binaries Computed at the AEI

\begin{tabular}{cccccccc}
\hline \hline Binary & $a$ & $\nu$ & $x / M$ & $\tilde{J}$ & $a_{\text {fin }}$ & $a_{\text {fin }}^{\text {fit }}$ & $\begin{array}{c}\mid \text { Error } \mid \\
(\%)\end{array}$ \\
\hline$t 8 \ldots \ldots \ldots$ & -0.5840 & 0.2500 & 3.1712 & 2.432 & 0.4955 & 0.4981 & 0.53 \\
$t a 8 \ldots \ldots$ & -0.3000 & 0.2500 & 3.7078 & 3.000 & 0.5941 & 0.5927 & 0.23 \\
$t b 8 \ldots \ldots$ & -0.8000 & 0.2500 & 3.8082 & 2.200 & 0.4224 & 0.4227 & 0.08 \\
$t b 8 l \ldots \ldots$ & -0.8000 & 0.2500 & 4.8600 & 2.400 & 0.4266 & 0.4227 & 0.92 \\
$p 1 \ldots \ldots$. & -0.8000 & 0.1580 & 3.2733 & 0.336 & 0.0050 & 0.0046 & 9.89 \\
$p 2 \ldots \ldots \ldots$ & -0.5330 & 0.1875 & 3.3606 & 1.872 & 0.2778 & 0.2794 & 0.57 \\
$p 3 \ldots \ldots$ & -0.2667 & 0.2222 & 3.4835 & 2.883 & 0.5228 & 0.5216 & 0.23 \\
\hline
\end{tabular}

Note. - The different columns contain the initial spin $a$, the symmetric mass ratio $\nu$, half of the initial separation $x / M=\frac{1}{2}\left(x_{1}-x_{2}\right)$, the dimensionless initial angular momentum $\tilde{J}=J /(\mu M)$, the numerical and fitted values for $a_{\text {fin }}$, and the corresponding relative error.

the orbital angular momentum. We next express $a_{\text {fin }}$ as a thirdorder polynomial of $\nu$ and $a$,

$$
\begin{aligned}
a_{\mathrm{fin}}= & s_{0}+s_{1} a+s_{2} a^{2}+s_{3} a^{3}+s_{4} a^{2} \nu+s_{5} a \nu^{2} \\
& +t_{0} a \nu+t_{1} \nu+t_{2} \nu^{2}+t_{3} \nu^{3} .
\end{aligned}
$$

Expression (1) is a lowest-order Ansatz. It intends to capture the behavior of a function known exactly only in the extreme mass-ratio limit (EMRL) and which has support from numerical simulations in two restricted regimes, i.e., $\nu=1 / 4,0 \leq$ $|a| \lesssim 0.75$ and $0.16 \leq \nu \leq 1 / 4, a=0$. A priori there is no reason to believe that the proposed fit $a_{\mathrm{fin}}(\nu, a)$ will capture the general behavior well, but in fact it does.

Given the available numerical estimates, it is possible to calculate the coefficients $s_{0}-S_{5}$ and $t_{0}-t_{3}$ by simply performing a two-dimensional (2D) least-squares fit to the data. This, however, would require a lot of care and is likely to lead to inaccurate estimates. This is because the space of parameters presently accessible to numerical simulations is rather small. Reliable results are in fact available only for spins $|a| \lesssim 0.8$ and mass ratios $q \equiv M_{2} / M_{1} \gtrsim 0.25$ and thus corresponding to $\nu \gtrsim 0.16$. However, it is possible to exploit exact results which hold in the EMRL, i.e., for $\nu=0$, to constrain the coefficients in expression (1). It is worth emphasizing that the EMRL results are not only exact, but also in regimes that numerical relativity simulations cannot probe. More specifically, we can exploit that in the EMRL the final spin cannot be affected by the infinitesimally small $\mathrm{BH}$. In practice, this amounts to requiring that

$$
a_{\text {fin }}(a, \nu=0)=a,
$$

which constrains four of the six coefficients:

$$
s_{0}=s_{2}=s_{3}=0, \quad s_{1}=1 \text {. }
$$

Additional but nonexact constraints can also be applied by exploiting the knowledge, near the EMRL, of the functional dependence of $a_{\text {fin }}$ on the mass ratio. A convenient way of doing this is suggested by BKL07, and within this approach we perform a Taylor expansion of $a_{\mathrm{fin}}$ for $\nu \ll 1$ and determine that

$$
\begin{gathered}
\left|a_{\text {fin }}^{\prime}\right|_{(a=1, \nu=0)}=2(\sqrt{3} / 3-1), \quad\left|a_{\text {fin }}^{\prime}\right|_{(a=0, \nu=0)}=2 \sqrt{3}, \\
\left|a_{\text {fin }}^{\prime}\right|_{(a=-1, \nu=0)}=2(1+19 \sqrt{15} / 45),
\end{gathered}
$$

where $a_{\mathrm{fin}}^{\prime} \equiv \partial a_{\mathrm{fin}} / \partial \nu$. The coefficients in equation (1) are then

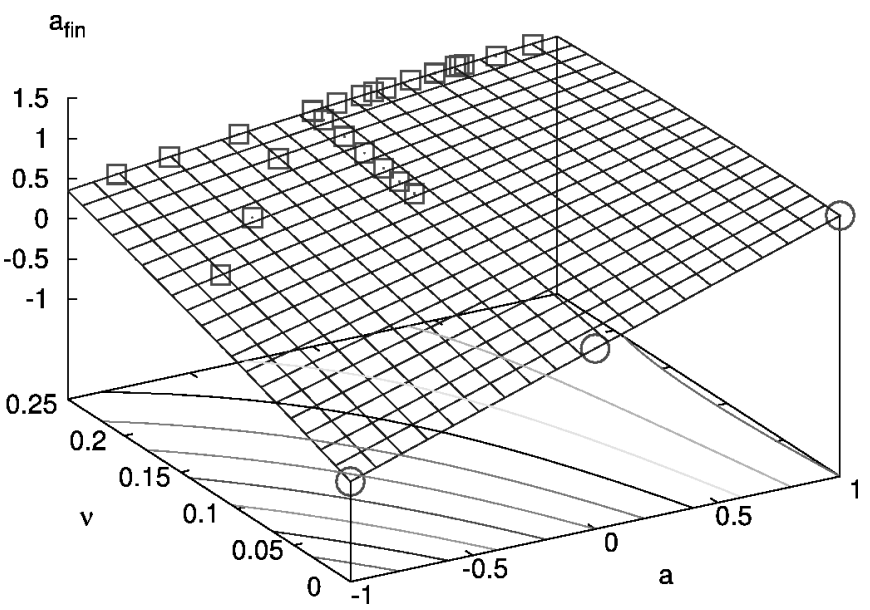

FIG. 1.-Global dependence of the final spin on the symmetric mass ratio and on the initial spins as predicted by expression (5). Squares refer to numerical estimates while circles to the EMRL constraints. [See the electronic edition of the Journal for a color version of this figure.]

$s_{4}=\sqrt{3}(19 \sqrt{5}-75) / 45, \quad t_{1}=2 \sqrt{3}, \quad$ and $\quad t_{0}=[\sqrt{3}(15-$ $19 \sqrt{5})-90] / 45$. While this may seem a good idea, it leads to bad fits to the data. We believe this is due to two distinct reasons: (1) the lack of accurate numerical data for near-extreme BHs, i.e., $|a| \approx 1$, which therefore leads to incorrect estimates of the coefficients; (2) expressions (4) are analytic but not exact and should be used with caution. There are, in fact, deviations from analyticity in $\nu$ as $\nu \rightarrow 0$, and as revealed by the presence of integer powers of $\nu^{1 / 5}$ during the transition between the last stable orbit and the plunge (see Buonanno \& Damour 2000). In the case of nonspinning binaries $(a=0)$, it is now possible to verify that the deviations are indeed very small (Damour \& Nagar 2007), but this check is not possible for very large spins. In view of this and to make the minimal number of assumptions, we retain the analytic estimate only for the coefficient $t_{1}$, so that equation (1) has five out of 10 coefficients constrained analytically,

$$
a_{\text {fin }}=a+s_{4} a^{2} \nu+s_{5} a \nu^{2}+t_{0} a \nu+2 \sqrt{3} \nu+t_{2} \nu^{2}+t_{3} \nu^{3} \text {. }
$$

Determining the remaining five coefficients from a leastsquares fit to the available data yields

$$
\begin{gathered}
s_{4}=-0.129 \pm 0.012, \quad s_{5}=-0.384 \pm 0.261, \\
t_{0}=-2.686 \pm 0.065, \quad t_{2}=-3.454 \pm 0.132, \\
t_{3}=2.353 \pm 0.548,
\end{gathered}
$$

with surprisingly small residuals and large error bars only for $s_{5}$. The functional behavior of expression (5) and the position of the numerical data points are shown in Figure 1.

In the following we discuss the properties of the proposed fit, providing evidence that it represents a very accurate description of the available estimates, and discuss how to use it to make astrophysically interesting predictions.

1. The estimate for the final spin in the case of equal masses and the comparison with available data and estimates is made in Figure 2. The top panel shows the numerical estimates (circles for the AEI data [Rezzolla et al. 2007] and stars for the FAU-Jena data [Marronetti et al. 2007]), the BKL07 estimate, and our 2D fit through equation (5). The bottom panel shows 


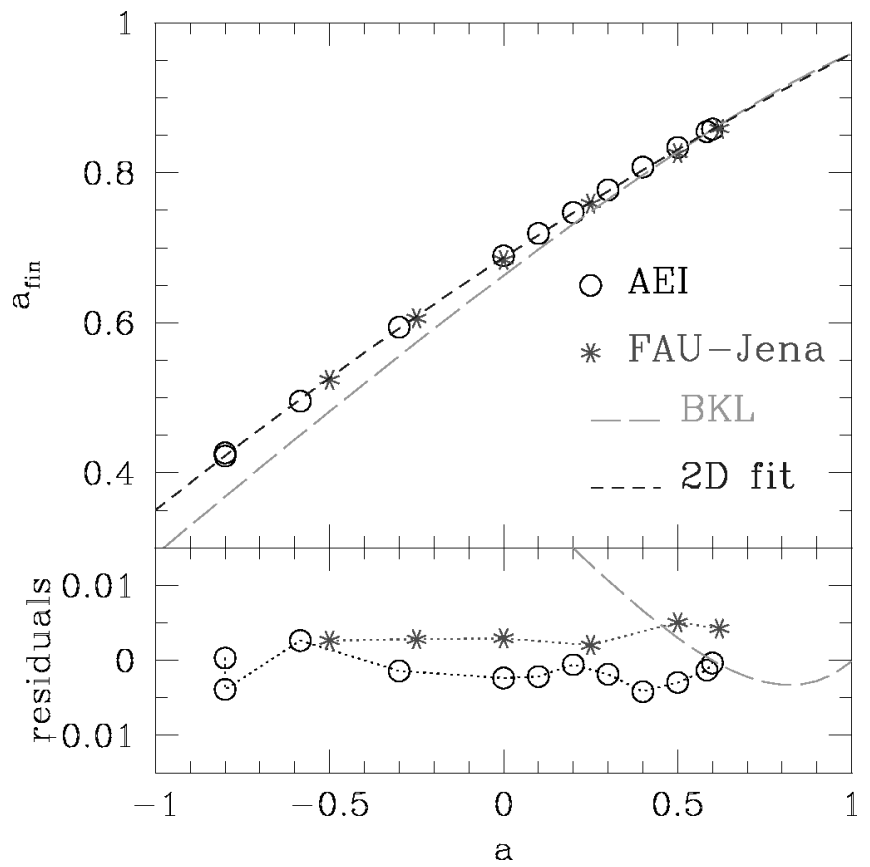

FIG. 2.-Top: Comparison of the numerical data with the 2D fit through eq. (5) in the case of equal-mass binaries, $(\nu=1 / 4)$. Open circles indicate the AEI data (Rezzolla et al. 2007), stars the FAU-Jena data (Marronetti et al. 2007), a long-dashed line the BKL07 data, and a short-dashed one the fit. Bottom: Residuals between the different estimates and the fit. [See the electronic edition of the Journal for a color version of this figure.]

the residuals between the different estimates and the 2D fit; these are always of a few percent only and become larger for the BKL07 estimate when $a \lesssim 0$.

2. Despite the cubic dependence assumed in equation (1), equation (5) is only quadratic with $a$. When $\nu=1 / 4$, it confirms what was obtained recently (Rezzolla et al. 2007), indicating that, for equal-mass binaries, the next order will be 4 .

3. Using equations (5) and (6) we estimate that the minimum and maximum final spins for an equal-mass binary are $a_{\text {fin }}=0.3502 \pm 0.03$ and $a_{\text {fin }}=0.9590 \pm 0.03$, respectively.

4. For nonspinning binaries, expression (5) is cubic in $\nu$, and a comparison with the available data and the estimate from the effective-one-body (EOB) approach combined with testmass limit predictions for the ring-down (Damour \& Nagar 2007) are shown in Figure 3. In particular, the top panel shows the numerical values (empty circles for the Jena data [Berti et al. 2007] and stars for the Goddard data [Buonanno et al. 2007b]), a long-dashed line for the quadratic EOB 1D fit (Damour \& Nagar 2007) and a short-dashed line for our 2D fit. (Because it is very similar to the EOB estimate, we have not shown the BKL07 prediction.) The residuals in are shown in the bottom panel.

5. A physically useful condition that can be deduced from the $2 \mathrm{D}$ fit is the values of the initial spin and mass ratio that will lead to a final Schwarzschild BH (Hughes \& Blandford 2003; BKL07). In practice this amounts to requiring $a_{\text {fin }}(a$, $\nu)=0$ in equation (5), and this curve in the $(a, \nu)$ plane is shown in the top panel of Figure 4. Binaries on the curve produce Schwarzschild BHs, while binaries above the curve start with a positive total angular momentum and end with a positive one; binaries below the curve, on the other hand, start with a positive total angular momentum and end with a negative one, i.e., with a global flip. Also shown in the top panel of Figure 4 is the prediction from BKL07: $\left.a_{\text {Schw }}\right|_{\text {BKL07 }}=$

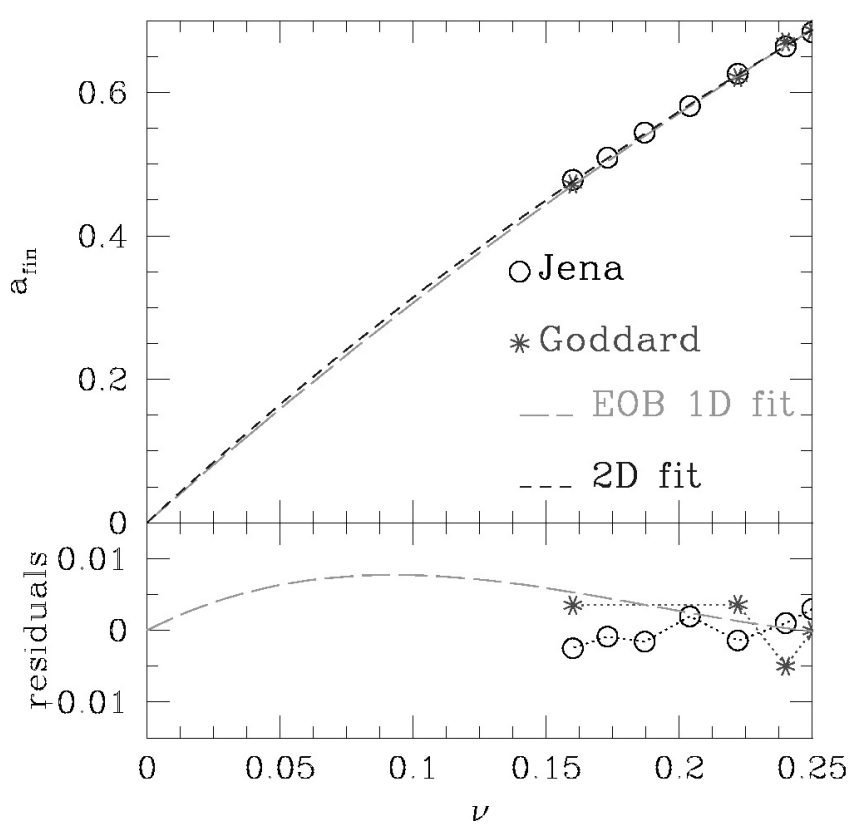

FIG. 3.-Top: Comparison of the numerical data with the 2D fit through eq. (5) in the case of nonspinning binaries. Open circles indicate the Jena data (Berti et al. 2007), stars the Goddard data (Buonanno et al. 2007b), a longdashed line the quadratic EOB fit (Damour \& Nagar 2007), and a short-dashed line our 2D fit. Bottom: Residuals between the different estimates and the 2D fit. [See the electronic edition of the Journal for a color version of this figure.]
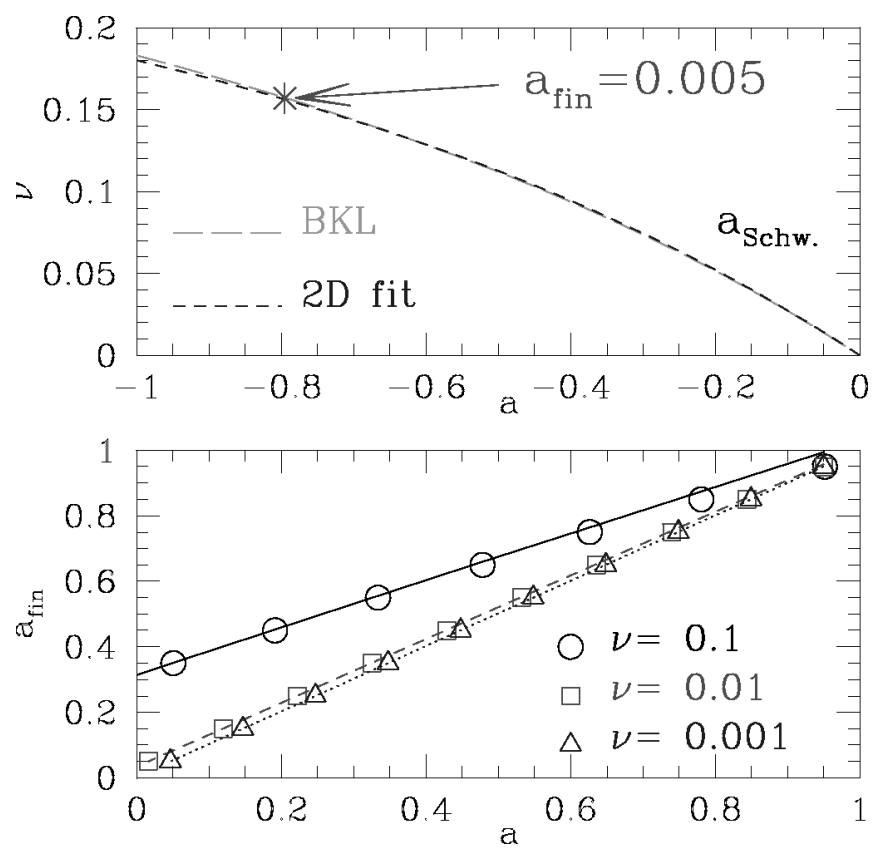

FIG. 4.- Top: Set of initial spins and mass ratios leading to a final Schwarzschild BH, i.e., $a_{\mathrm{fin}}(a, \nu)=0$. The two curves refer to the BKL07 estimate (long-dashed curve) and to the 2D fit (short-dashed curve). Indicated with a star is a numerical example leading to $a_{\text {fin }}=0.005$. Bottom: Comparison between the BKL07 prediction (symbols) and the 2D fit (solid, dashed, and long-dashed lines) near the EMRL. Different curves refer to different values of $\nu$ and the match is complete for $\nu=0$. [See the electronic edition of the Journal for a color version of this figure.] 
$2 \nu \sqrt{3} /(2 \nu-1)$. The two estimates are very similar for all values of $\nu$ and small differences appear for $\nu \gtrsim 0.15$, where the BKL07 estimate is less accurate. Shown with a cross is the binary $p_{1}$ (see Table 1), which yields a final $\mathrm{BH}$ with spin $a_{\text {fin }}=0.005$. The numerical value is between the BKL07 prediction and the 2D fit.

6. The BKL07 estimate is expected to be particularly accurate for $\nu \ll 1$, and its prediction in this regime is captured very well by the $2 \mathrm{D}$ fit (of course, the two predictions are identical for $\nu=0$ ). This is shown in the bottom panel of Figure 4 with different curves referring to $\nu=0.001,0.01$, and 0.1 ; interestingly, the differences are small even for $\nu=$ 0.1 .

7. It is simple to derive the value of $a$ which will produce a final $\mathrm{BH}$ with the same spin as the initial ones. This amounts to requiring that $a_{\text {fin }}(a, \nu)=a$ in equation (5), and the resulting solution is shown in Figure 5; clearly, the axis $\nu=0$ is a trivial solution and a magnification of the behavior away from the EMRL is shown in the inset. For equal-mass binaries the critical value is $a_{\text {crit }}=0.9460$, in very good agreement with the BKL07 estimate $a_{\text {crit }} \gtrsim 0.948$. The minuteness of the region for which $a_{\text {fin }}<a$ (dashed region) suggests that BHs are typically spun up by mergers (Hughes \& Blandford 2003).

8. It is easy to verify that by setting $\nu=1 / 4$ and $2 a=$ $a_{1}+a_{2}$ in equation (5), the coefficients $s_{1}-s_{5}$ and $t_{0}-t_{3}$ coincide, within the error bars, with the coefficients $p_{0}, p_{1}$, and $p_{2}$ reported in Rezzolla et al. (2007) for equal-mass, unequal-spin binaries. The fact that the fit here is equivalent to, but has been independently derived from, the one for the equal-mass, unequalspin binaries, is an indication of its robustness. Indeed, it is possible to extend equation (5) to the whole $\left(a_{1}, a_{2}, \nu\right)$ space, i.e., to describe the final spin of generic aligned, unequal-spin, unequal-mass $\mathrm{BH}$ binaries, by replacing $a$ with $\left(a_{1}+\right.$ $\left.a_{2} q^{2}\right) /\left(1+q^{2}\right)$. The resulting expression reduces to equation (5) for unequal-mass, equal-spin binaries, and to the one in Rezzolla et al. (2007) for equal-mass, unequal-spin binaries. Our suggested extension of equation (5) to the $\left(a_{1}, a_{2}, \nu\right)$ space is the simplest one which recovers, for aligned spins, the welltested limits of equal mass, unequal spins and unequal mass, equal spins. Work is in progress to validate thisAnsatz with numerical simulations.

A final comment is one of caution. The dependence of the final spin on the mass ratio in the case of extreme aligned $\mathrm{BHs}$ is particularly challenging to calculate and has not yet been investigated accurately by numerical calculations. The predictions of expression (5) in this limit amount to mere extrapolations and are therefore accurate to a few percent at most. As an example, when $a=1$, the fit given in expression (5) is a non-monotonic function with maximum $a_{\text {fin }} \simeq 1.029$ for $\nu \simeq$ 0.093; this clearly is an artifact of the extrapolation.

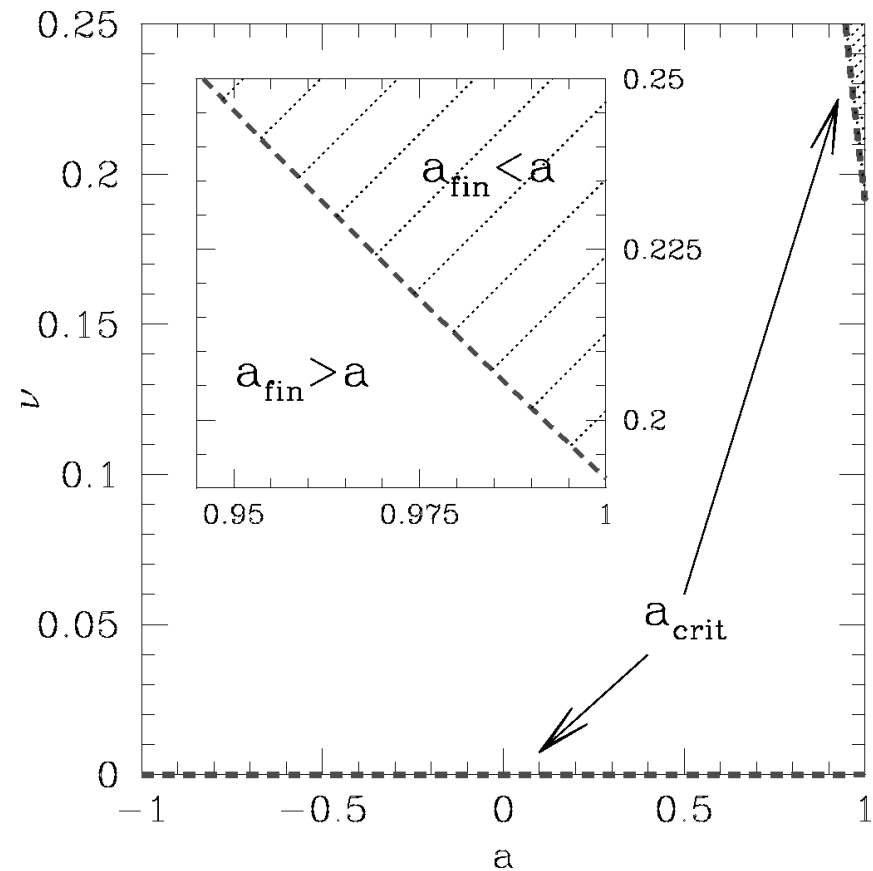

FIG. 5.-Critical values of the initial spin and mass ratio leading to a final $\mathrm{BH}$ having the same spin as the initial ones, i.e., $a_{\mathrm{fin}}(a, \nu)=a$. A magnification is shown in the inset, where the dashed (nondashed) region refers to binaries spun down (spun up) by the merger. [See the electronic edition of the Journal for a color version of this figure.]

\section{CONCLUSIONS}

Modeling the final spin in a generic binary $\mathrm{BH}$ merger is not trivial given the large space of parameters on which this quantity depends. We have shown that the results of recent simulations combined with basic but exact considerations derived from the EMRL allow us to model this quantity with a simple analytic expression in the case of $\mathrm{BH}$ binaries having unequal masses and unequal spins which are aligned with the orbital angular momentum. When compared with all other estimates coming either from numerical calculations or from approximation techniques, the estimates of the $2 \mathrm{D}$ fit show differences which are of few percent at most.

We thank A. Buonanno, T. Damour, S. A. Hughes, L. Lehner, A. Nagar, and B. S. Sathyaprakash for discussions. We are grateful to D. Merritt for pointing out an error in the interpretation of our results. The computations were performed on the supercomputers at AEI, LITE, LSU, LONI, and NCSA. Support comes also through the DFG grant SFB/TR 7.

\section{REFERENCES}

Berti, E., et al. 2007, Phys. Rev. D, 76, 064034

Boyle, L., Kesden, M., \& Nissanke, S. 2007, preprint (arXiv: 0709.0299)

Buonanno, A., Chen, Y., \& Damour, T. 2006, Phys. Rev. D, 74, 104005

Buonanno, A., \& Damour, T. 2000, Phys. Rev. D, 62, 064015

Buonanno, A., Kidder, L., \& Lehner, L. 2007a, preprint (arXiv:0709.3839) (BKL07)

Buonanno, A., et al. 2007b, Phys. Rev. D, 76, 104049

Campanelli, M., et al. 2007, Phys. Rev. D, 75, 064030

Damour, T. 2001, Phys. Rev. D, 64, 124013
Damour, T., \& Nagar, A. 2007, Phys. Rev. D, 76, 044003

Gonzalez, J. A., et al. 2007a, Phys. Rev. Lett., 98, 091101 Herrmann, F., et al. 2007, Phys. Rev. D, 76, 084032

Hughes, S. A., \& Blandford, R. D. 2003, ApJ, 585, L101

Koppitz, M., et al. 2007, Phys. Rev. Lett., 99, 041102

Marronetti, P., et al. 2007, preprint (arXiv:0709.2160)

Pollney, D., et al. 2007, Phys. Rev. D, 76, 124002

Rezzolla, L., et al. 2007, preprint (arXiv:0708.3999) 